Journal of the Magnetics Society of Japan Vol. 15 Supplement, No. S2 (1991)

(C) 1991 by The Magnetics Society of Japan

\title{
EDDY CURRENT AND DISPLACEMENT CURRENT ANALYSIS FOR MULTI-LAYER HEAD MATERIALS
}

\author{
Shinji TANABE, Hiroshi OHJI, Kazunori INOUE and Tatsuo OZEKI
}

Materials \& Electronic Devices Laboratory, Mitsubishi Electric Corporation, 8-1-1 Tsukaguchi-Honmachi, Amagasaki, Hyogo 661, Japan

Abstract--- Some analytical and experimental results of displacement current and eddy current in multi-layer soft magnetic films are discussed in this paper. As a result using a 3D-FEM (Finite Element Method) with a time dependence term, it was made clear that the order of the displacement current is smaller than that of the eddy current by $10^{6}$. The reason of the permeability decline at higher frequency in multi-layer films was not in the displacement current but in the electric conductivity along the film edges. A high permeability double layer thick film with small eddy current losses was obtained by removing the covered metal along the film edge.

\section{INTRODUCTIION}

As the recording densities of the nagnetic recording are increasing, the signal frequency has become higher. Eddy current loss has become one of the significant problems in the development of soft magnetic films for MIG(Metal-In-Gap) heads or thin film heads.

In order to decrease the eddy current loss, it is said that multi-layered films are effective in thick soft magnetic films[1]. However, there was a hypothesis that displacement current flows through the insulator such as a capacitor sandwiched by two metal films and the effect of depositing a multi-layered film is not so efficient at a high frequency. There are no reports to investigate the effect of the displacement current systematically by theories and experiments. In the real multi-layer films, the effect of separating the thick film by insulators has not confirmed experimentally.

We tried to estimate the order of the displacement current between two metal films using a 3-D FEM including a time dependent term. In order to confirm the effect of the separation by a insulator, a sample with no metal coverage along the insulator edges were fabricated and the permeability was measured.
THEORY

Calculation method

A three dimensional finite element method (FEM) including a time dependent term $(\partial B / a t)$ was used in the calculations of eddy currents and displacement currents. The eddy current values was computed directly by the FEM program. In order to obtain the displacement current values between the metal films separated by an insulator, the distribution of the eddy current in the insulator was computed at first. Using this current density $j$ distribution and the insulator's conductivity $\sigma$, the electric field E distribution is induced by the following equation:

$$
E=j s / o
$$

where $s$ is the finite element area and $d$ is the distance between the two metal films separated by an insulator. From this electric field distribution and the insulator's dielectric constant, the electric flux density is calculated:

$$
D=E E
$$

The displacement current jd is;

$$
j d=\partial D / \partial t=D \cdot \omega
$$


Table 1. Properties and sizes of a model

\begin{tabular}{|c|c|c|c|c|c|}
\hline & $\bar{\mu}$ & $\bar{\varepsilon}$ & $\begin{array}{c}\sigma \\
(1 / \Omega \mathrm{m})\end{array}$ & $\begin{array}{c}\text { Thickness } \\
(\mu \mathrm{m})\end{array}$ & $\begin{array}{c}\text { Area } \\
\left(\mu \mathrm{m}^{2}\right)\end{array}$ \\
\hline $\begin{array}{c}\text { MAGNETIC } \\
\text { FILM }\end{array}$ & 1000 & 1 & $10^{6}$ & 2 & $20 \times 20$ \\
\hline INSULATOR & 1 & 3 & - & 0.5 & $20 \times 20$ \\
\hline
\end{tabular}

The total displacement current is;

$$
\iint j d \cdot d s
$$

FEM mode1

In order to consider the eddy current, the $\mathrm{T}-\Omega$ method [2] was used in the 3-D FEM computation. Fig. 1 shows a $1 / 8$ model for the eddy current loss and displacement current analyses of a doublelayered film. In this model an insulation film is sandwiched between two metal soft magnetic films. $10 \mathrm{MHz}$ sinusoidal $\mathrm{AC}$ magnetic field is applied from the side of the film as shown in Fig.1. The geometry and properties of the films are indicated in Table 1.

Calculation results

Fig. 2 shows a distribution of the eddy current on a cross-section plane of a

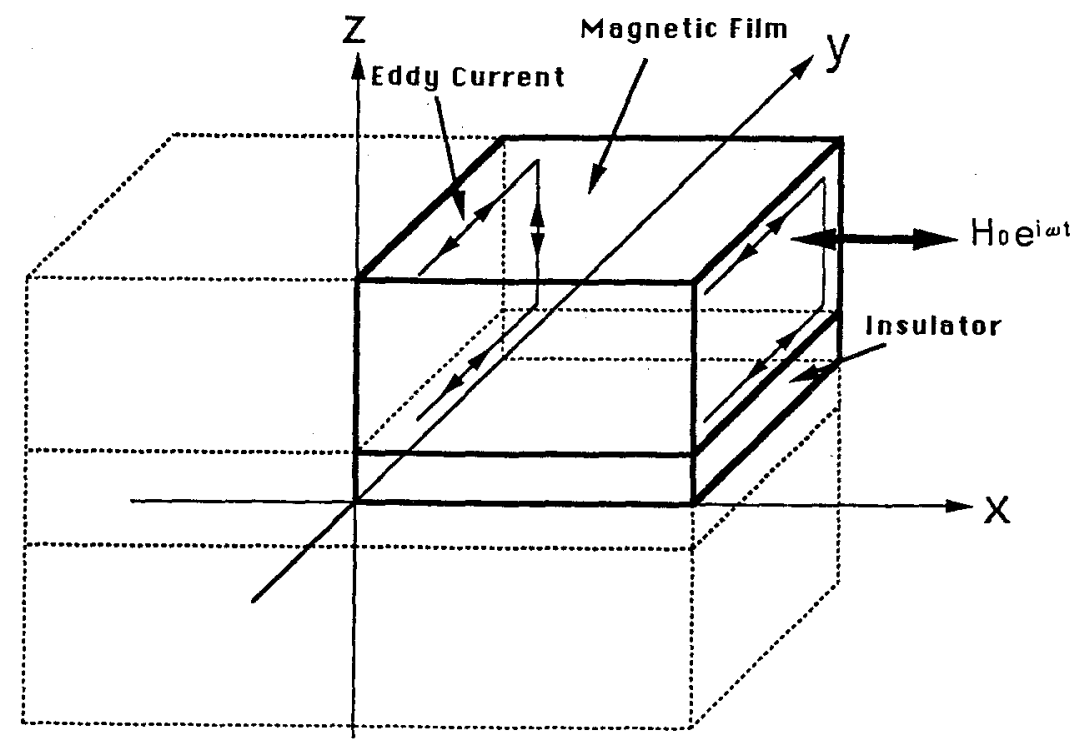

Figure 1. Calculation model for eddy and displacement current 
(a)

A

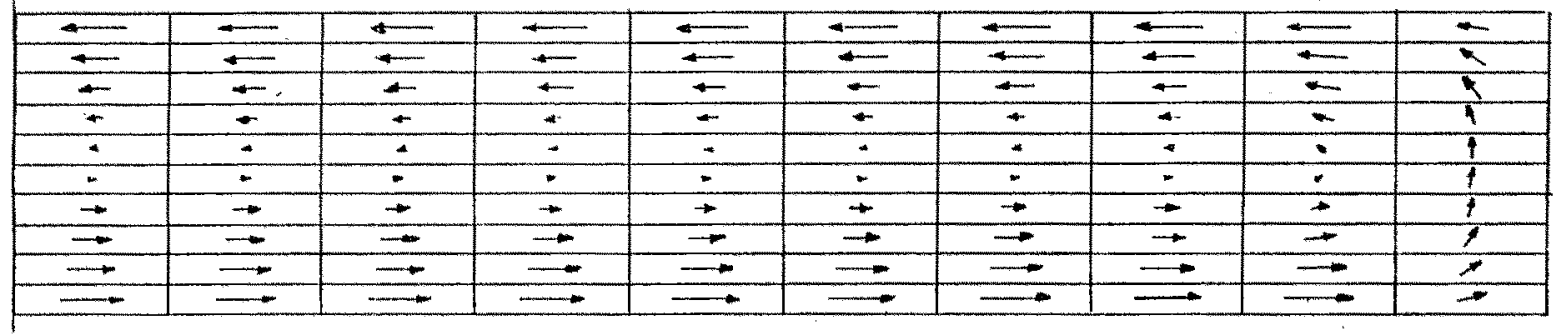

C

B

(b)
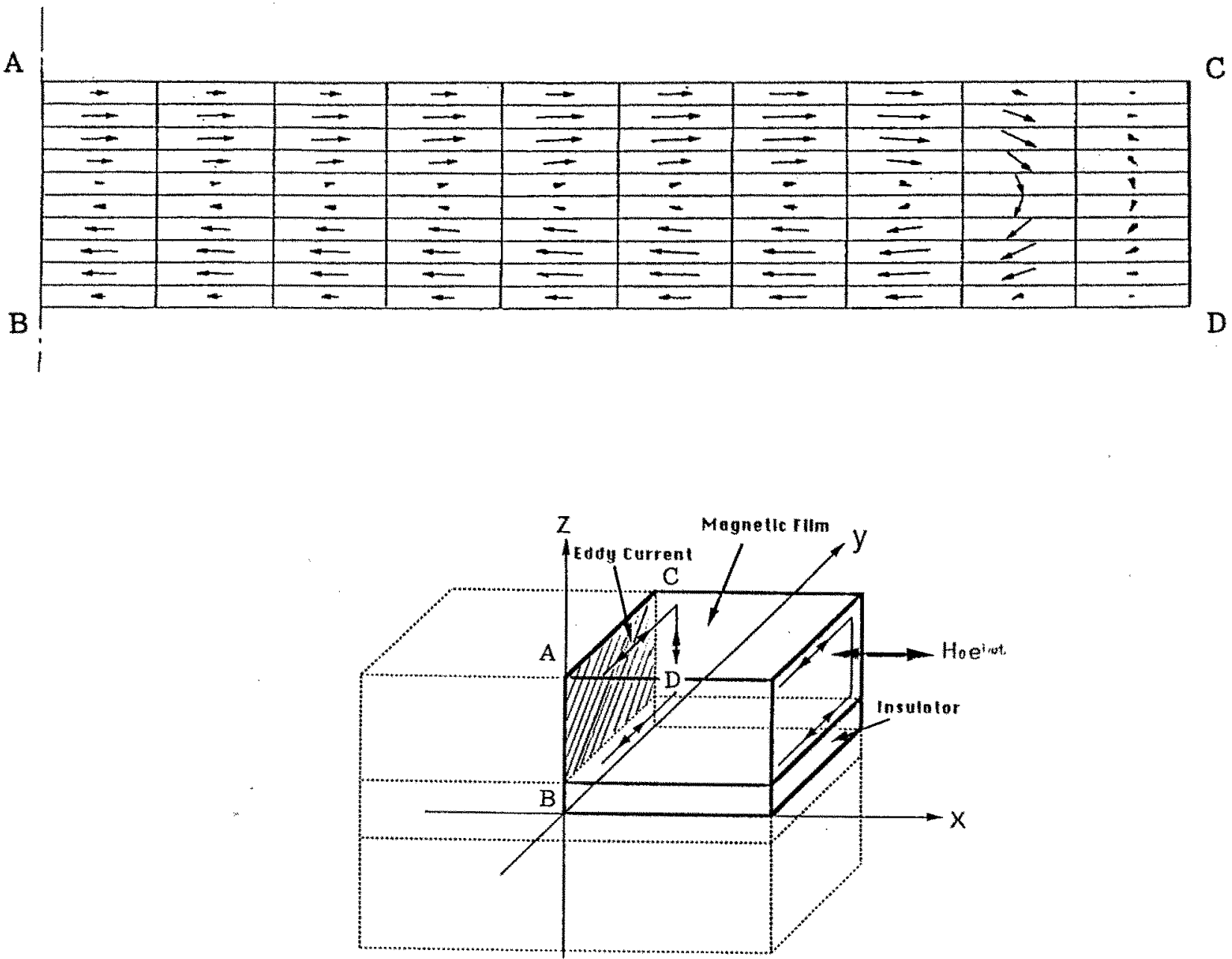

Figure 2. Eddy current in a magnetic film. (a) 90 degrees different phase current from the applied magnetic field. (b) The same phase current as the applied magnetic field. 

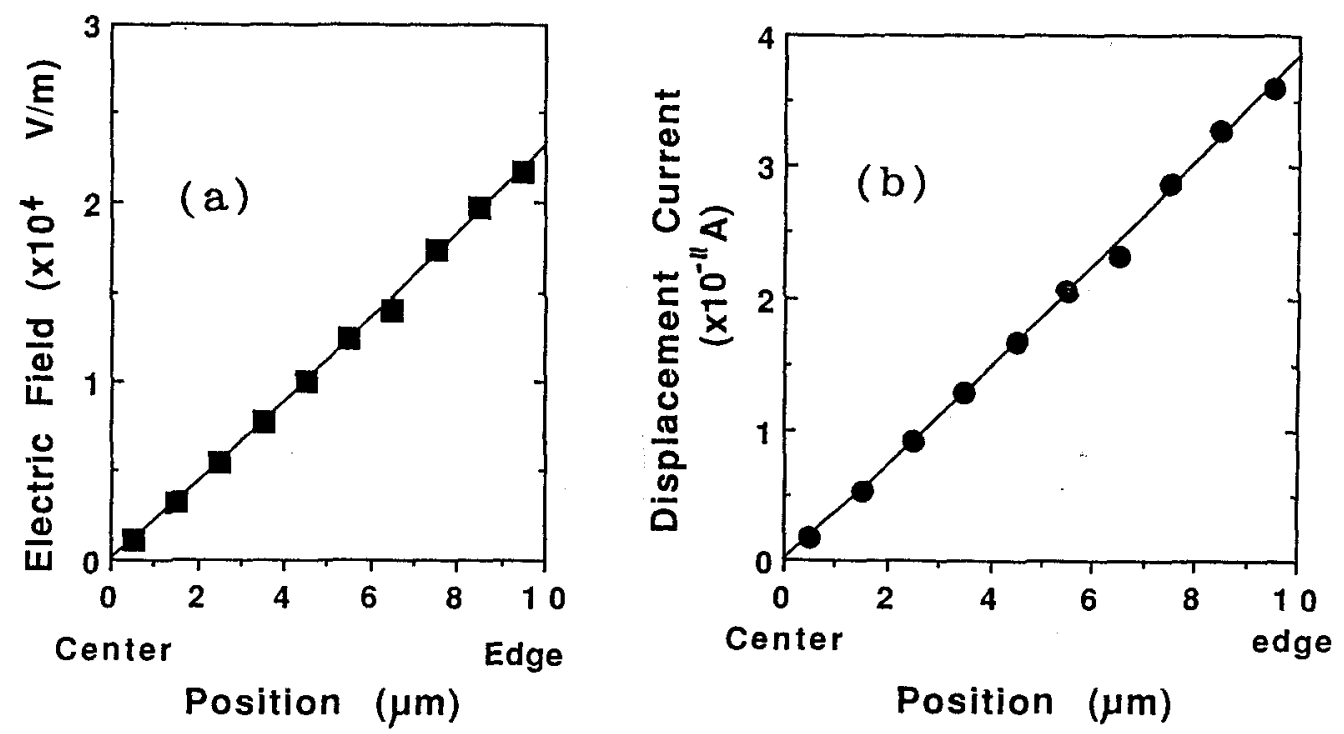

Figure 3. (a) Electric field distribution along the centerline of the model. (b)Displacement current distribution between two metals unit finite element area.

magnetic film. (a) is the eddy current of the 90 degree different phase from the applied magnetic head. (b) is the eddy current in phase of the applied field. Fig. 3 shows the electric field distribution along the center line of the model. The displacement current at $10 \mathrm{MHz}$ AC field was calculated from this distribution using (2) and (3). The maximum electric field in a finite element was about $2.0 \times 10^{4}[\mathrm{~V} / \mathrm{m}]$ and the electric flux density becomes $5.3 \times 10^{-7}\left[\mathrm{c} / \mathrm{m}^{2}\right]$. The displacement current through a $1 \mathrm{um}^{2}$ finite element area was only $10^{-11}$ [A]. While, the maximum value flowing through a $1 \mu^{3}$ cubic finite element in the metal film was about $5.0 \times 10^{-4}[\mathrm{~A}]$. The result is shown in Fig.3(b). The order of the displacement current is smaller than the eddy current by $10^{6}$. As a conclusion, it was made clear that the displacement current can be ignored in the range of $5 \mathrm{MHz}$ to $50 \mathrm{MHz}$ and separating a thick soft magnetic layer is an effective method to prevent an eddy current loss.

\section{EXPERIMENTS}

We made it clear that the separation

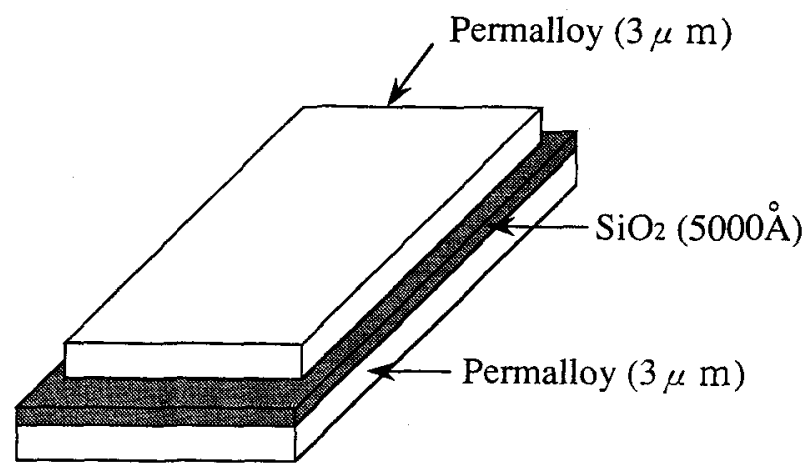

Figure 4. Sample 2's schematic geometry.

of a thick film by an insulator is effective for decreasing the eddy current losses in the above theoretical approach. However, the effect of multi-layering a film has not reported experimentally. The reason was supposed that the metal materials are covered over the edges of the insulator. In order to prove this assumption, we tried following experiments. 
Samples

Three types of double layer samples were fabricated for this experiment.

1) Samples just sputtered on substrates

2) Samples that the upper magnetic layer is a little bit smaller than the insulator and the lower magnetic layer as shown in Fig. 4 .

3) Samples that the edge side was lapped to remove the covered metal materials.

The geometry of the samples and the materials are shown in the Table 2. As the magnetic films, 3 um and 6 um permally films were used. As the insulator between the lower and upper magnetic films, 5000A Sio2 film was used.

Results

Fig. 5 shows the permeability properties as a function of the magnetic field frequency for 3 and $6 \mu \mathrm{m}$ single-layer films. The theoretical lines were calcu-

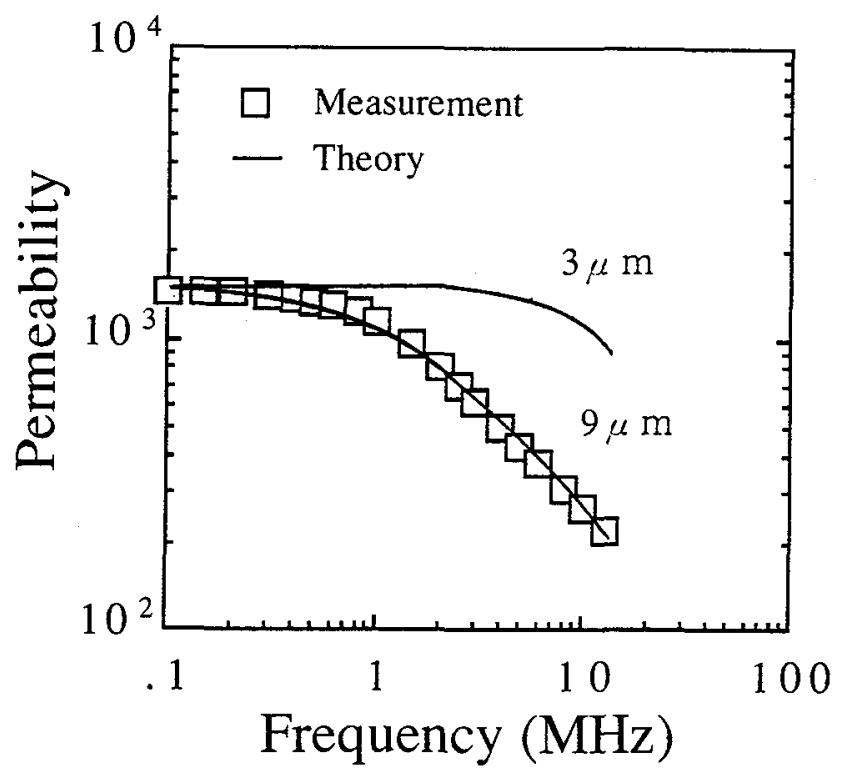

Figure 6. Permeability properties as a function of the frequency for $9 \mu \mathrm{m}$ ordinary triple-layer (sample 1). Each magnetic layer thickness is $3 \mu \mathrm{m}$.

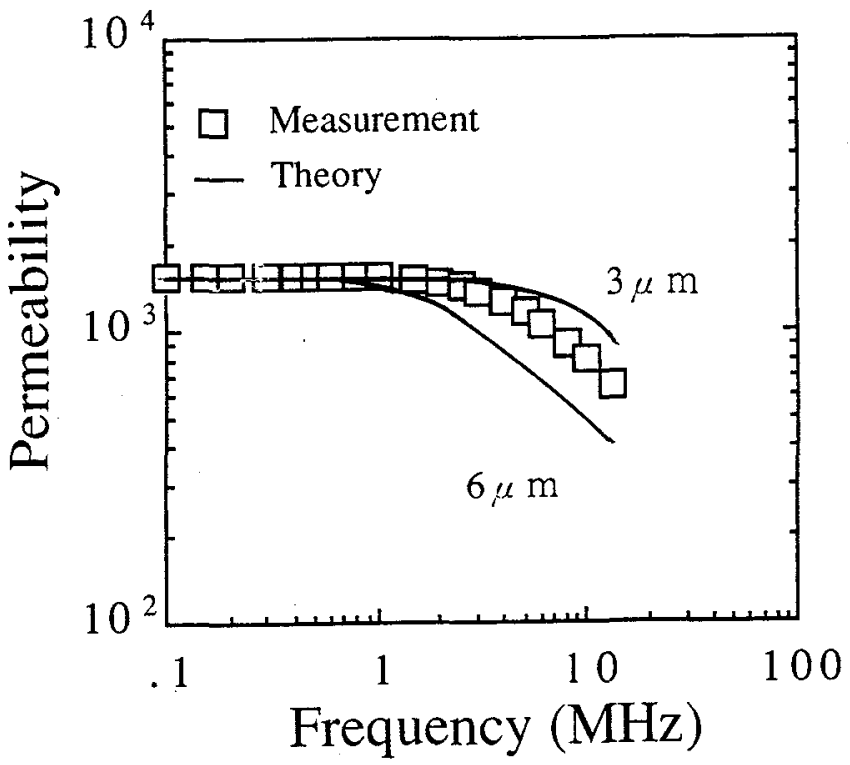

Figure 7. Permeability properties as a function of the frequency for $6 \mu \mathrm{m}$ double-layer (sample 2). Each magnetic layer thickness is 3 um.
Figure 5. Permeability properties as a function of the frequency for 3 and 6 um single-layer films.

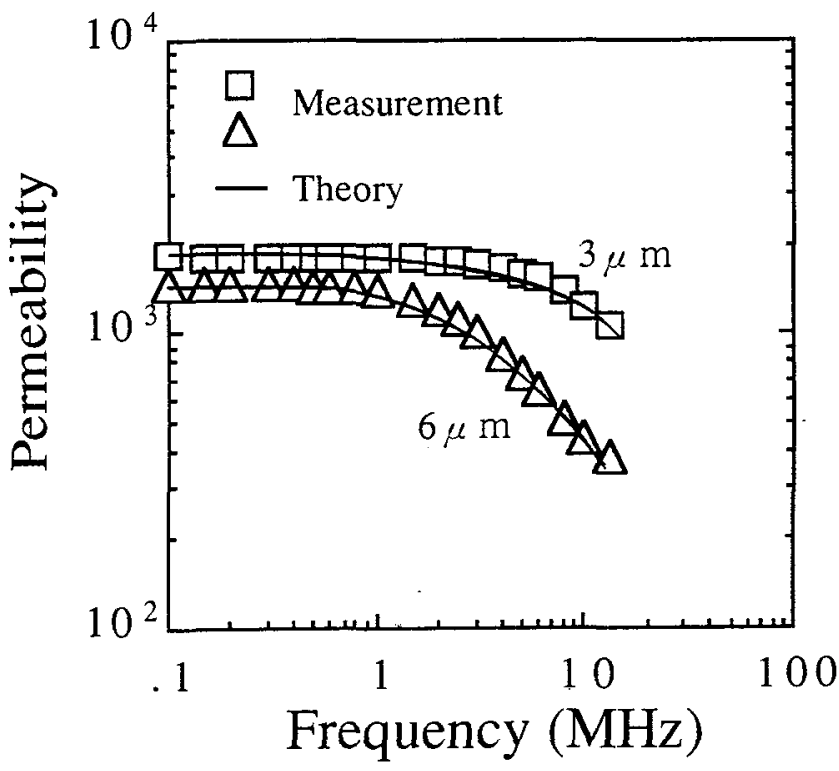


lated using analytical equations induced by Bozorth [3]. The measured values agree with the theoretical lines very well. As shown in Fig.6, no effects of the separation by an insulator appear in the sample (1). While, for samples (2) and (3), significant effects of the separation appear as shown in Fig.7. The main difference between the sample (1) and samples $(2),(3)$ is along the edge of the insulator. In case of the sample (1), metal films were covered along the edges of the insulator. Therefore, it is assumed that an eddy current flow through this covered metal freely. This eddy current is the reason that the permeability seems to be declined as same as the $6 \mu \mathrm{m}$ single layer films even though this film is separated into two $3 \mu \mathrm{m}$ films by an insulator. While, there are no electric contact along the edges of the insulator in samples (2), (3). This is the reason that the frequency properties of permeability were improved almost as same as the $3 \mu \mathrm{m}$ thick film's ones. However, the properties were little bit worth than the $3 \mu \mathrm{m}$ thick film's ones. This decline seems to be caused by an eddy current through pin holes of the insulator or the diffusion of metals.

\section{CONCLUSIONS}

In case of double or multi-layer films, the order of the displacement current between two metal films is enough smaller than the eddy current by the order of $10^{6}$. Therefore, the reason that the effect of the separation of a thick film by an insulator was not observed is not in the displacement current but in the eddy current pass through the covered metals along the insulator edges. By fabricating double-layer samples without covered metal along the insulator edges, the $6 \mu \mathrm{m}$ thick films frequency properties of permeability was improved almost as same as that of the two $3 \mu \mathrm{m}$ films properties. From those results, it was made clear that the geometry of film edges is important to decrease the eddy current losses for thin film heads and laminated heads.

\section{ACKNOWLEDGMENTS}

The authors would like to express their thanks to Dr. Shibata for his helpful encouragements.

\section{REFERENCES}

[1]J.P.Lazzari and I.Melnick,"Integrated magnetic recording heads,"IEEE Trans. Magn., Vol. MAG-7, no. 1, pp.146-150, Mar. 1971

[2]T.Tanaka, S.Nakamura and K.ogura, The Institute of Electrical Engineers of Japan, SA-88-33, pp.49-58 (In Japanese)

[3]R.M.Bozorth "Ferromagnetism, Chapter $17 "$ pp. 769-810 